\title{
A STUDY OF EASY MAGNETIZATION AXES OF FERRO- NANOPARTICLES ON MAGNETIC HYPERTHERMIA
}

\author{
Abu-Bakr A.F. ${ }^{1}$, Zubarev A.Yu. ${ }^{1}$ \\ 1) Institute of Natural Sciences and Mathematics, Ural Federal University, \\ Ekaterinburg, 620083, Russia \\ E-mail: alibakrm@yahoo.com
}

We interest to show the role of Neel relaxation magnetization and magnetization axes of particles in the present work. Theoretical study and the mathematical modeling are formulated on the basis of remagnetization dynamics (Debye equation) and the intensity of heat production (hyperthermia effect).

This work deals with theoretical study and mathematical modeling of the easy magnetization axes of particles on the one of biomedical applications $[1,2]$ that called magnetic hyperthermia treatment. Magnetic hyperthermia is a new type of cancer treatment allowing to achieve selective heating of a tumor without damaging of the surrounding healthy tissues. The main idea of this method is in embedding of ferromagnetic nanoparticles in a tumor region and heating them with the help of alternating magnetic field. In the temperature corridor $42-50 \mathrm{C}$ the tumor cells undergo protein destruction, which leads to the cells death, whereas the cells of the healthy tissue, being more temperature resistant, are not injured. Magnetic hyperthermia is a way of tissue heating, by using thermal effect produced by embedded magnetic nanoparticles under the action of oscillating magnetic field.

There are two main mechanisms of heat production by a ferromagnetic particle placed in an alternating magnetic field. The first mechanism is due to the energy viscous dissipation at the particle rotation in a carrier medium. The second mechanism corresponds to Neel relaxation of the particle magnetic moment. Depending on the particle size and mechanical properties of the carrier medium, either the first or the second mechanism dominates (see, for example, [3]). We interest to show the role of Neel relaxation magnetization and magnetization axes of particles in the present work. The mathematical modeling is formulated on the basis of remagnetization dynamics (Debye equation) and the intensity of heat production (hyperthermia effect). The system occurs under external magnetic field. The obtained models can be solved analytically and numerically and compared with the previous studies. Analysis of results shows that the mechanism of Neel relaxation and easy magnetization axes of particles on magnetic hyperthermia. This fact should be taken into account at the organization of biomedical hyperthermia therapy.

1. Rosensweig R.E. J. Magn. Magn. Materials, 252, 370 (2002).

2. Abu-Bakr A.F., Zubarev A.Yu. J. Magn. Magn. Materials. 477, 404 (2019).

3. Goya G.F., Lima E., Torres T.E., Rossi L.M.. IEEE Trans. Mag. 44, 4444 (2008). 